\title{
sciendo
}

RESEARCH PAPERS FACULTY OF MATERIALS

SCIENCE AND TECHNOLOGY IN TRNAVA

SLOVAK UNIVERSITY OF TECHNOLOGY

IN BRATISLAVA

2019, Volume 27, Number 44

DOI 10.2478/rput-2019-0013

\section{SAFETY CONCERN OF NANOMATERIALS}

\author{
Alica BARTOŠOVÁ ${ }^{1}$, Tatyana NIKOLAEVNA IVANOVA ${ }^{2}$ \\ ${ }^{1}$ SLOVAK UNIVERSITY OF TECHNOLOGY IN BRATISLAVA, \\ FACULTY OF MATERIALS SCIENCE AND TECHNOLOGY IN TRNAVA, \\ INSTITUTE OF INTEGRATED SAFETY, \\ Ulica JÁna Bottu 2781/25, 91724 TRNAVA, SLOVAK REPUbliC \\ ${ }^{2}$ TCHAIKOVSKY BRANCH PERM NATIONAL RESEARCH POLYTECHNIC INSTITUTE \\ 617764 LENIN ST., 73, TCHAIKOVSKY, RUSSIA \\ email: tatnic2013@yandex.ru, alica.bartosova@stuba.sk \\ Received: 17.05.2019, Accepted: 14.06.2019, Published: 25.07.2019
}

\begin{abstract}
Nanomaterials are sure to take an important part in shaping the 21 st century. Nevertheless, their increasing application in many fields of the industry also turns attention to the impact on health and environment. To achieve this goal, it is necessary to fully understand the influence of nanoparticles on the environment and the relevant safety issues.

The aim of the present review is to characterise the current state of regulation and legislation of nanomaterials, as well as the factors of safety assessment and their toxicity.
\end{abstract}

\section{Key words}

Nanomaterials, nanoparticles, safety, regulations, toxicity

\section{INTRODUCTION}

Over the last years, the field of nanotechnology has spurred several major advances in science, engineering, and manufacturing since the industrial revolution. Nanotechnology is the technology dealing with both single nanoobjects and materials, and devices based on them, and with the processes that take place in the nanometer range. Nanomaterials are those materials whose key physical characteristics are dictated by the nanoobjects they contain. Nanomaterials are classified into compact materials and nanodispersions. A nanoparticle (NMs) is a quasizero-dimensional (0D) nanoobject, in which all characteristic linear dimensions are of the same order of magnitude (not more than $100 \mathrm{~nm}$ ) [1].

According to the origin, NMs could be classified as natural or synthetic NMs. Natural nanomaterials are produced in nature through (bio)geochemical or mechanical processes, without direct or indirect connection to human activity or anthropogenic process. Synthetic or engineered (ENMs) are conceived, designed, and intentionally produced by humans or nanomaterials unintentionally produced as a result of any form of direct or indirect human influence or anthropogenic process (incidental nanomaterials) [2]. Photochemical reactions, 
volcanic eruptions, and forest fires are some of the natural processes that lead to the production of natural NPs [3].

For categorization of nanoparticles, there are some important criteria, such as dimensionality and composition. Nanoparticles have dimensions in the range 1-100 $\mathrm{nm}$ and may exist as 1D (deposited layer on a solid surface), 2D (nanowires or nanotubes attached to a large surface, or nanopores in a solid surface or membrane) and 3D objects ("single" particles, such as wires, nanotubes, spheres, etc.) [4].

Nanomaterials can be organized into four groups according to their base:

- carbon-based nanomaterials - generally, these NMs contain carbon and they can be found as tubes, ellipsoids, and spheres. Fullerenes, carbon nanotubes, carbon nanofillers, graphene belong to this group [3],

- inorganic-based nanomaterials - these NMs contain metal or metal oxide, they can be synthetized and modified with various chemical functional groups.

- organic-based nanomaterial - these NMs made mostly from organic matter and have application in the biomedical field as a drug carrier and delivery. For example, lipid-based nanoparticle (Liposomes), protein-based NPs or micelles [5],

- composite-based nanomaterials - these NMs are multiphase NPs and nanostructured materials (NSMs) with one phase on the nanoscale dimension that can either combine NPs with other NPs or NPs combined with larger or with bulk-type materials (e.g., hybrid nanofibers) or more complicated structures, such as a metal-organic framework. The composites may be any combinations of carbon-based, metal-based, or organic-based NMs with any form of metal, ceramic, or polymer bulk materials [3].

The usage of nanomaterials (NMs) is expected to have great potential to improve consumer and industrial products, address critical energy needs, enhance security systems, and improve the medical field [6]. There are many nanomaterials used as ingredients in pharmaceutical, cosmetics, food, in an industry for their unique physical properties such as magnetic, optical, mechanical, electrical properties, and quantum mechanics.

Despite the benefits of integrating NMs into many fields of industry, pharmaceutical or food, there are concerns about the potential impact on health and safety. Currently, there is a knowledge gap between technological progress and nano-safety research. However, as with any new technology or material, the identification of potential health risks is a prerequisite for a proper assessment of the usefulness and safety of the new chemicals, materials, and products that may be developed.

\section{REGULATION}

The EU's regulatory framework generally covers nanomaterials. Legislation on specific consumer products (Cosmetic Products, Novel Foods, Biocidal Products, Medical Devices) addresses nanomaterials, including requirements for information on nanomaterials (labeling) and assessment of the safety of these materials. Other regulations implicitly apply to nanomaterials, e.g. the chemicals regulation $\mathrm{REACH}$, as the regulations addresses all chemicals including nanomaterials. However, due to their novel and/or nanospecific properties and often different behaviour as compared to "ordinary" chemicals, there are uncertainties about the safety of nanomaterials and its proper assessment.

Today, there are several pieces of legislation in the European Union (EU) with specific references to NMs. However, a single internationally accepted definition for NMs does not exist. According to the Environmental Protection Agency (EPA), "NMs can exhibit unique properties dissimilar than the equivalent chemical compound in a larger dimension. The International Organization for Standardization (ISO) has described NMs as a "material with 
any external nanoscale dimension or having the internal nanoscale surface structure". Likewise, the term nanomaterial is described as "a manufactured or natural material that possesses unbound, aggregated or agglomerated particles where external dimensions are between 1-100 $\mathrm{nm}$ size range", according to the EU Commission [3, 7]. The EC NM (The European Commission's Recommendation on a definition of the term 'nanomaterial') definition further specifies:

- fullerenes, graphene flakes and single wall carbon nanotubes with one or more external dimensions below $1 \mathrm{~nm}$ should be considered as nanomaterials,

- 'particle', 'agglomerate' and 'aggregate' are defined as follows:

a) 'particle' means a minute piece of matter with defined physical boundaries;

b) 'agglomerate' means a collection of weakly bound particles or aggregates where the resulting external surface area is similar to the sum of the surface areas of the individual components;

c) 'aggregate' means a particle comprising of strongly bound or fused particles [8].

Nanomaterials are not necessarily hazardous; therefore, the EC NM definition is not based on hazard or risk assessment. It cannot differentiate between hazardous and non-hazardous materials as neither particle size nor specific surface area can be directly linked to hazard. It simply identifies, according to well-defined criteria, a certain group of materials as 'nanomaterial', which might deserve specific considerations in a regulatory context [8].

The EC NM definition refers to natural, incidental and manufactured materials. It therefore covers a potentially very large number of materials, regardless of whether these are new and man-made for a specific purpose, unintentional by-products of human activity, or whether they naturally occur in the environment.

In order to reduce uncertainties regarding the safety of nanomaterials, best practices, guidelines and assessment practices, and methods for the safety testing of nanomaterials are being developed, e.g. by ECHA and within the OECD test guidelines programme. Still, more research with specific relevance for regulatory questions is needed, in particular regarding the implementation of the definition of nanomaterials, the enforcement of product labeling for the presence of nanomaterials and the development of methods for the safety testing of nanomaterials.

On 3 December 2018, the Commission adopted Commission Regulation (EU) 2018/1881 to modify REACH Annexes I, III and VI-XII, introducing nano-specific clarifications and new provisions in the chemical safety assessment (Annex I), registration information requirements (Annex III and VI-XI) and downstream user obligations (Annex XII). The proposed amendments that will enter into mandatory application by 1/1/2020 for the new as well as the existing registrations will significantly clarify $\mathrm{REACH}$ registration requirements with regard to nanomaterials.

\section{ASPECT OF SAFETY ASSESSMENT OF NANOMATERIALS}

As for risk assessment and risk management of nanomaterials, the characterization and identification of anticipated risks should be first determined for chemical substances or foods. For the investigation of the hazard potential of nanomaterials, specific test systems observing the effects on humans and the environment are recommended. Further, the ECHA requires a realistic scenario for exposure, including a detailed description of the production process, uses of the product through the whole life-cycle, a concept for human and environmental exposure control and specific environmental release categories (SPERCs). SPERCs characterize a material by defining exposure levels based on "good practice" and "professional use", according to [9] they have become an essential part of environmental safety assessment [9].

A risk assessment consists of four components: 
1. Hazard identification: a qualitative evaluation of the adverse effects of a substance;

2. Exposure assessment: evaluation of the types (routes and media) and magnitude or levels of exposure;

3. Dose-response evaluation: the relationship between dose and incidence (or severity) of an adverse effect (e.g., noncancerous and cancer); and

4. Risk characterization: quantitative estimation of the probable incidence of adverse health effects under various conditions of exposure, including a description of the uncertainties involved [10].

Important is the determination of physical and chemical properties (e.g. size, shape, solubility) that affect the potential toxicity of nanoparticles, assessing the short-term and longterm effects that nanomaterials can have on organs and tissues (e.g. lungs), determination of the biological (physiological) potential toxic effects, the establishment of the first and subsequent medical assistance models for assessing potential risks, determining the units of measurement appropriate for the determination of the toxicity of a given nanomaterial.

Clearly, characterizing every physicochemical parameter of test material will be impractical for most toxicological studies. Given the wide range of analytical techniques available in many disciplines associated with nanotechnology, multidisciplinary collaborations with research and analysis groups offering state-of-the-art nanomaterial characterization capabilities are strongly recommended when conducting toxicological studies with nanomaterials. In vitro studies, which have become an essential component of risk assessmentdirected research paradigms for chemicals, pharmaceuticals, consumer products, and fine and ultrafine particulates, are an essential element of all tiered approaches for toxicity assessment of nanomaterials that have been proposed. These studies allow specific biological pathways or toxicological endpoints to be tested under controlled conditions, as well as the isolation of mechanistic pathways that is not feasible in vivo.

\section{Particle size and surface area}

NPs show size-dependent toxicity. There are several methods applied to determine particle size qualitatively and quantitatively. They include laser diffraction dynamic light scattering, static light scattering and nanoparticle tracking analysis (NTA), SEM and TEM. In combination with separation technologies, e.g. size exclusion chromatography or field flow fractionation, they are applicable to a wide spectrum of materials in the nanometer and the micrometer range [9].

Typically, the toxicological effect of NPs increases with decreasing particle size and increasing surface area. It can also be noted that nano and microparticles of the same mass dose react differently with the human cells [3]. The physisorption method, BET is a reliable method for the surface area determination. The BET theory can be derived similar to the Langmuir theory, but by considering multilayered gas molecule adsorption, where it is not required for a layer to be completed before an upper layer formation starts [11].

Nanoparticles have been surface conjugated for applications for various uses. Some surface conjugations cause more grave cytotoxicity than others. Therefore, relations between surface conjugations and their safety for cells have to be considered [12].

\section{Agglomeration or aggregation}

In case of agglomeration, a group of nanoparticles held together by relatively weak forces (van der Waals, electrostatic or surface tension) and aggregate means that a heterogeneous particle in which the various components are held together by relatively strong forces not easily broken apart. Information on the aggregation and agglomeration of a nanomaterial can be used to predict the ability of absorption translocation and distribution in the body, which can be used 
in the selection of relevant internal barrier models and relevant cell types for in vitro assays [13]. For determination of these characteristics, TEM, SEM, AFM (Atomic force microscopy) or DLS (Dynamic light scattering) can be used [10].

\section{Delivered dose}

The dose is defined as the amount or quantity of a substance that will reach a biological system. The dose is directly related to the exposure or the concentration of a substance in the relevant medium (air, food, water) multiplied by the duration of contact. Attention should be paid to the tendency of nanomaterials to agglomerate, as that can have a serious impact on factors like dilution, internalised dose, and application of assessment factors. High dose can give results not representative for lower concentrations due to agglomeration and subsequent lower biological uptake, biological barrier penetration, or sedimentation affecting the internal exposure or in cases where effect mechanisms do not scale with concentrations [14].

\section{Dissolutions}

Dissolution is the key element to identify whether a nanomaterial is stable enough to exert nanospecific behaviour. It is very important to know if a nanomaterial dissolves into its molecular or ionic form and how fast, where and under which circumstances this takes place. Dissolution is a critical factor linked to bioaccessibility/bioavailability. The solubility of material also greatly influences the cytotoxicity of different nanoparticles. The rate of dissolution is dependent on a particle's chemical and surface properties, and size and is further influenced by the surrounding media. The nanoparticles potential to dissolve influences their existence in the environment and controls their biological response [13].

\section{Reactivity}

Concerns about the health implications of nanomaterials have been expressed because particles and materials in the nano-size range may pose toxicological hazards due to their enhanced reactivity (e.g., chemical, electrical, and magnetic). In general, the greater chemical reactivity of nanomaterials results in increased production of reactive oxygen species (ROS), including free radicals and it is one of the primary mechanisms of nanoparticle toxicity; it may result in oxidative stress, inflammatory cytokines production and consequent damage to proteins, membranes, DNA and cell death [15].

\section{Exposure routes}

Nanoparticles can either be deliberately introduced into the body for medical purposes (drug delivery systems) or absorbed involuntarily from the environment (inhalation of nanoparticle-containing dust in the air). Inhalation is the main route of exposure to nanoparticles followed by the dermal and oral routes of exposure during nanomaterial production or product formulation. Particles inhaled with the air through the mouth and nose pass through the throat [12] [10]. Therefore, analytical methods such as imaging techniques (e.g., scanning electron microscopy [SEM] or transmission electron microscopy [TEM], dynamic light scattering) may have to be employed to identify the shape and chemical nature of the nanomaterials. However, many of these techniques are associated with specialized research tools and may be very expensive and are not necessarily practical for routine exposure monitoring in the workplace.

\section{Surface modification - coating}

Nanoparticles have been coating for applications for various uses. The surface manipulation makes the different physical, chemical, or biological characteristics than the one originally presented on the particle surface which can be induced natural, positive, or negative charge onto the particle surface. Particle surface plays a critical role in toxicity as it makes 
contact with cells and biological material. Surface coatings can render noxious particles nontoxic while less harmful particles can be made highly toxic. For example, according to [16] nanoscale zerovalent iron (nZVI) modified by bismuth caused strong toxic effects on all of the tested organisms, except for earthworm.

\section{TOXICITY}

The small size of NPs facilitates translocation of active chemical species from organismal barriers such as the skin, lung, body tissues and organs. Nanoparticles primarily enter the human body through inhalation or dermal contact, and secondarily by injection or ingestion, if nanoparticles are present in medicines or foods [17]. Thus, irreversible oxidative stress, organelle damage, asthma, and cancer can be caused by NPs depending on their composition. The general acute toxic effects caused by exposure to NPs and nanostructured materials include reactive oxygen species generation, protein denaturation, mitochondrial disconcertion and perturbation of phagocytic functions. Uptake by the reticuloendothelial system, nucleus, neuronal tissue and the generation of neoantigens that causes possible organ enlargement and dysfunction are common chronic toxic effects of NPs [3].

Some nanoparticles have been shown to be toxic titanium dioxide nanoparticles $\left(\mathrm{TiO}_{2} \mathrm{NPs}\right)$ have been known to pose threats to humans, livestock, and the ecosystem since being used in drug delivery systems, antibacterial materials, cosmetics, sunscreens, and electronics. The International Agency for Research on Cancer (IARC) has classified $\mathrm{TiO}_{2} \mathrm{NPs}$ to be a Group 2B carcinogen for humans based on its exposure [17]. Table 1 referres to the application and potential toxicity of chosen NPs.

\begin{tabular}{|c|c|c|}
\hline Nanoparticles & Applications & Potential Toxicity effects \\
\hline Quantum dots & $\begin{array}{l}\text { Imaging, diagnosis, electronic } \\
\text { applications }\end{array}$ & Inflammation, liver damage \\
\hline Gold & $\begin{array}{l}\text { Photodynamic cancer therapy, } \\
\text { radiotherapy, gene therapy }\end{array}$ & $\begin{array}{l}\text { liver damage, activation of } \\
\text { hepatic macrophages }\end{array}$ \\
\hline Silver & Drug delivery, diagnosis, imaging & $\begin{array}{l}\text { Kidney damage, inflammation, } \\
\text { mineralization }\end{array}$ \\
\hline Liposomes & Drug/protein/gene delivery & inflammation \\
\hline $\mathrm{TiO}_{2}, \mathrm{ZnO}$ & $\begin{array}{l}\text { Paints, cements, sunscreen, car } \\
\text { cosmetics, catalysts, UV-protection, } \\
\text { batteries }\end{array}$ & $\begin{array}{l}\text { Cytotoxicity, cell membrane } \\
\text { damage, and increased } \\
\text { oxidative stress }\end{array}$ \\
\hline CNT & $\begin{array}{l}\text { Electronics, additive to tyres, } \\
\text { lubricants, used in adsorption of } \\
\text { contaminants }\end{array}$ & DNA damage, \\
\hline Iron oxide & $\begin{array}{l}\text { Biomedical, drug delivery, and } \\
\text { diagnostic fields }\end{array}$ & $\begin{array}{l}\text { Oxidative stress and DNA } \\
\text { damage }\end{array}$ \\
\hline
\end{tabular}

There are many unknowns as to whether the unique properties of engineered nanomaterials pose health concerns. The potential health risk following exposure to a substance is generally associated with the following:

- magnitude and duration of the exposure,

- persistence of the material in the body,

- inherent toxicity of the material,

- susceptibility or health status of the person. 
There is a whole multidisciplinary field of science - Nanotoxicology focusing on determining the extent to which nanomaterials pose a hazard to human health and the environment. It encompasses the physicochemical determinants, routes of exposure, biodistribution, molecular determinants, toxicity, and regulatory aspects of nanomaterials. By studying the toxicological potential of nanomaterials, data may be obtained that will be useful for conducting future safety and risk assessments for these novel substances and for products that contain them.

It is important to develop a balance between the development of nanomaterials and the conduct of research necessary to identify potential health hazards and to acquire critical data for conducting future safety assessments of nanomaterials and the products that incorporate them.

\section{CONCLUSION}

More specific data on nanomaterials are required to address regulatory needs, to account for the specificities of nanomaterials, and to assess their safety. The basic components of risk assessment of chemicals are hazard and exposure assessments, dose-response estimation, risk characterisation, and accounting for uncertainty in the overall assessment.

Properties of nanomaterials can be different from of the non-nanoform of the same material. They might pose a risk to human health and the environment which is different to the non-nanoform. Safety data generated on the non-nanoform therefore are not mandatory adequate for the nanomaterilas. Moreover, tests which have been developed for the safety assessment of chemicals might not be (directly) applicable to nanomaterials, or even completely new tests might be required that capture the peculiarities of nanomaterials. Guidelines for safety assessment of chemicals will have to be adapted to fully capture possible hazards and risks originating from the use of nanomaterials. In light of the uncertainties regarding the safety of nanomaterials, it is necessary to develop best practices, facilitate harmonization of assessment practices and methodologies, and to increase confidence and mutual understanding among stakeholders how to best approach the risk assessment of nanomaterials.

Beyond doubts, nanoparticles pose threat to the ecosystem and human beings. It will be dangerous to rely on isolated reports on biocompatibility and safety of NPs to justify the uncontrolled use and applications of them.

\section{References}

[1] GUBIN, S. P. 2009. Magnetic Nanoparticles. WILEY-VCH Verlag GmbH \& Co, 2009. ISBN 9783-527-40790-3

[2] HPCHELLA, M. et al. 2019. No. 6434 Natural, incidental, and engineered nanomaterials and their impacts on the Earth system, Science (80-. )., 363, 1-10. ISSN 1095-9203

[3] JEEVANANDAM, et al. 2018. Review on nanoparticles and nanostructured materials : history, sources, toxicity and regulations. Beilstein J. Nanotechnol., 9, 1050-1074. ISSN 21904286

[4] DIBENEDETTO, A., FASCIANO, S.,COLUCCI, A. 2013. Nanosized particles : questioned for their potential toxicity, but some are applied in biomedicine. In: Inorganic Micro- and Nanomaterials - Synthesis and Characterization, De Gruyter. ISBN 978-3-110306-66-8

[5] KUMAR, R., LAL, S. 2015. Molecular Nanotechnology Synthesis of Organic Nanoparticles and their Applications in Drug Delivery and Food Nanotechnology: A Review, J. Nanomater. Mol. Nanotechnol.. ISBN 9621329477

[6] SANTAMARIA, A., SAYES, C. M. 2010. Risks ToxicologicaI Studies with Nanoscale Materials. Nanotechnology Environmental Health and Safety. Elsevier Inc., ISBN 9780128135891

[7] GOTTARDO, et al. 2017. NANoREG framework for the safety assessment of nanomaterials, EUR 28550 EN. doi 10.2760/245972. 
[8] RAUSCHER, H. et al. 2019. An overview of concepts and terms used in the European Commission 's definition of nanomaterial. Chem. Ing. Tech. Wiley-VCH Verlag GmbH \& Co. 224-231 ISSN $1522-2640$

[9] WACKER, M. G., PROYKOVA, A., SANTOS, G. M. L. 2016. Dealing with nanosafety around the globe - Regulation vs. innovation. Int. J. Pharm., 509(1-2), 95-106. ISSN 1873-3476

[10] SAYES,C. M., DUVAL, A. L., SANTAMARIA, A. B. 2018. Consumer Products Containing Nanomaterials: Toxicological Attributes and Exposure Potential. Nanotechnology Environmental Health and Safety, Elsevier Inc. ISBN 9780128135891

[11] NDOLOMINGO, M. J., MEIJBOOM, R. 2016. Determination of the surface area and sizes of supported copper nanoparticles through organothiol adsorption - Chemisorption. Appl. Surf. Sci., 390, 224-235. ISSN 0169-4332

[12] YAMAMOTO, K. 2018. Safety Assessment for the Nanoparticles. Nanoparticle technology handbook. Elsevier, pp. 380-400. ISBN 9780444641106

[13] DEKKERS, S. et al. 2016. Towards a nanospecific approach for risk assessment. Regul. Toxicol. Pharmacol., 80, 46-59. ISSN 10960295

[14] OOMEN, A. G. et al. 2018. Risk assessment frameworks for nanomaterials: Scope, link to regulations, applicability, and outline for future directions in view of needed increase in efficiency. NanoImpact, 9, No. July 2017, 1-13. ISSN 2452-0748

[15] RAMAKRISHNA, D., RAO, P. 2011. Nanoparticles :Is toxicity a concern? EJIFCC, 22, 92-101. ISSN 1650-3414

[16] YOON, H. et al. 2018. Ecotoxicology and Environmental Safety Impact of surface modification on the toxicity of zerovalent iron nanoparticles in aquatic and terrestrial organisms. Ecotoxicol. Environ. Saf., 163, No. November 2017, 436-443. ISSN 0147-6513

[17] BYSTRZEJEWSKA-PIOTROWSKA, et al. 2009. Nanoparticles: Their potential toxicity, waste and environmental management. Waste Manag., 29(9), 2587-2595. ISSN 0169-4332

\section{ORCID}

Alica Bartošová 0000-0003-1442-9652 